Special Issue of the 8th International Advances in Applied Physics and Materials Science Congress (APMAS 2018)

\title{
Conductive Polymer Doped with 2D-MoS 2 Coatings for the Corrosion Protection of Mild Steel
}

\author{
G. $\operatorname{ASAN}^{a}, \mathrm{~A} \cdot \mathrm{ASAN}^{b, *}$ AND H. ÇELIKKAN ${ }^{c}$ \\ ${ }^{a}$ Hitit University, Vocational School of Technical Science, Çorum, Turkey \\ ${ }^{b}$ Hitit University, Faculty of Engineering, Department of Chemical Engineering, Çorum, Turkey \\ ${ }^{c}$ Gazi University, Faculty of Science, Department of Chemistry, Ankara, Turkey
}

\begin{abstract}
In recent years, due to environmental and ecological concerns, electropolymerization of conductive polymers on non-noble metals has become important. In this study, adherent and homogeneous polyaniline and polypyrrole films doped with two dimensional molybdenum disulphide (2D-MoS 2 ) were electropolymerized onto mild steel (MS) in $0.1 \mathrm{M} \mathrm{H}_{2} \mathrm{C}_{2} \mathrm{O}_{4}$ by cyclic voltammetry method. The coatings obtained on the MS are characterized by SEM. The corrosion performance of polypyrrole, polyaniline, polypyrrole doped with $2 \mathrm{D}-\mathrm{MoS}_{2}$ and polyaniline doped with $2 \mathrm{D}-\mathrm{MoS}_{2}$ film coated on MS has been measured in sulfuric acid medium by the Tafel polarization method. The polypyrrole exhibited good corrosion protection properties. The polypyrrole coating has higher corrosion performance than polyaniline and polyaniline doped with $2 \mathrm{D}-\mathrm{MoS}_{2}$. The polypyrrole doped with $2 \mathrm{D}-\mathrm{MoS}_{2}$ showed the best corrosion performance in those coatings. This shows that polypyrrole $+2 \mathrm{D}-\mathrm{MoS}_{2}$ on $\mathrm{MS}$ has more stability and an impermeable layer against corrosion in the sulphuric acid solution.
\end{abstract}

DOI: 10.12693/APhysPolA.135.931

PACS/topics: corrosion, mild steel, two dimensional molybdenum disulphide, conductive polymers, coating

\section{Introduction}

Since DeBerry and Mengoli, who electropolymerized the aniline on stainless steel and iron, conductive polymers have begun to be used against corrosion $[1,2]$. In recent years, due to environmental and ecological concerns, chromating and phosphating of surfaces are undesirable. The discovery of the conducting polymers electrochemically synthesized as polypyrrole (PPy) [3], polythiophene [4] and polyaniline (PAN) [5, 6] bring an alternative process for the protection of oxidizable metals against the corrosion. Most of investigations of these conducting materials are mainly based on the electropolymerization of their monomers on noble metals or inert materials. However, the anodic dissolution of working electrode before attaining the oxidation potential of the monomer constitutes the main problem for the electrodeposition of polymers on oxidizable metals. Therefore, it is necessary to find new electrochemical conditions for slowing down electrode dissolution without preventing electropolymerization. Electropolymerization of pyrrole, aniline and thiophene on iron and steel surfaces has been performed in aqueous medium in the presence of oxalate as supporting electrolyte. The oxalate counter ion slows down the iron dissolution by leading to the formation of a passivation layer on the working electrode surface, and the electropolymerization starts on [7]. Conductive polymers are the best candidates due to their easy synthesis and their electrically conductivity for materials exposed to corrosion. The coating thickness and coating structure

*corresponding author; e-mail: asanabdurrahman@hotmail.com can be adjusted easily by changing the current density at the potential. In addition, it can be coated with different electrochemical methods such as potentiostatic, galvanostatic and cyclic voltammetry [8]. PPy and PAN are the most widely used conductive polymers for the protection of metals from corrosion. PAN is being used more than PPy for protection of metals corrosion. Because it has better environmental stability, easier synthesis and lower cost. These conductive polymers can be called "smart protective materials" because of their ability of store and transport charge. For this special feature, they are highly effective against corrosion [9].

In this study; firstly, the $\mathrm{MoS}_{2}$ layers were separated into two dimensional 2D-MoS $\mathrm{M}_{2}$ [10]. Later pyrrole, aniline, pyrrole with $2 \mathrm{D}-\mathrm{MoS}_{2}$ and aniline with $2 \mathrm{D}-\mathrm{MoS}_{2}$ have been electro polymerized on (MS) in oxalic acid by cyclic voltammetry method. The effects of these coatings have been investigated about corrosion of MS by the Tafel polarization method in sulfuric acid medium.

\section{Experimental procedure}

The experiments were carried out in a three-necked electrochemical cell with a capacity of $250 \mathrm{~mL}$. The solutions were prepared by the use of deionized water and Analar grade oxalic acid and sulfuric acid (98\%). The solutions prepared for the coating were prepared by adding the conductive polymer monomer (pyrrole or aniline) and $2 \mathrm{D}-\mathrm{MoS}_{2}$ into the 0.1 molar oxalic acid solution. As a result, coating solutions containing $0.1 \mathrm{M}$ oxalic acid $+0.1 \mathrm{M}$ conductive polymer monomer $+0.1 \mathrm{M}$ $2 \mathrm{D}-\mathrm{MoS}_{2}$ were prepared. The (MS) working electrode were immersed in to the solution from the middle neck. The surface of the electrode open to the solution was $0.5 \mathrm{~cm}^{2}$ for MS electrode. The electrode was embedded 
in a cylindrical shaped polyester resin with an external diameter of $1.0 \mathrm{~cm}$. Prior to each experiment, the working electrode was polished with a sandpaper of 4.000 in order to clean the surface of the working electrode, then cleaned by passing it through pure water and immersed in ethyl alcohol to accelerate the drying. The reference and counter electrodes were saturated calomel (SCE) and $1 \mathrm{~cm}^{2}$ platinum plate respectively. All the potentials are measured according to standard calomel electrode (SCE). The reference electrode was taken within the vicinity of the working electrode by the help of the tip of a lugging capillary to a distance equal to its diameter. The voltammetric measurements were made with Ivium CompactStat Instruments system. All cyclic voltammetry curves were obtained at a scan rate of $100 \mathrm{mV} / \mathrm{s}$ and the Tafel polarization curves at a scan rate of $2 \mathrm{mV} / \mathrm{s}$.

\section{Results and discussion}

PPy and PAN are electropolymerized on MS by cyclic voltammetry method in $0.1 \mathrm{M}$ oxalic acid. Figure 1 shows the trajectories of the electropolymerization obtained for 10 runs. It is visible, that effective electropolymerization of pyrrole starts at about $0.6 \mathrm{~V}$.

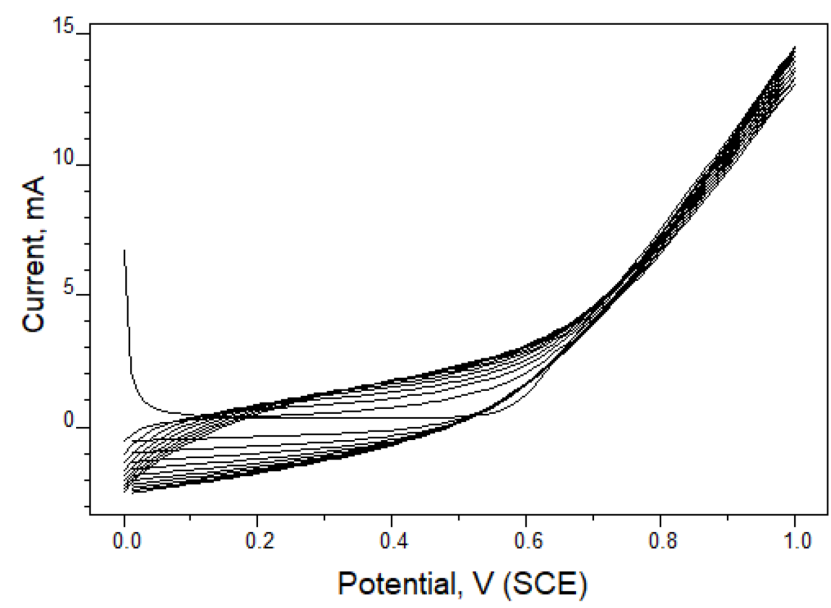

Fig. 1. Electropolymerization of pyrrole in $0.1 \mathrm{M}$ oxalic acid.

The corrosion rate was determined by the Tafel polarization method. The protection efficiency was estimated using the following expression, where $C R_{\text {uncoated }}$ and $C R_{\text {coated }}$ corrosion rate with and without coating, respectively.

$$
\eta=\frac{C R_{\text {uncoated }}-C R_{\text {coated }}}{C R_{\text {uncoated }}} \times 100 \%
$$

Figure 2 shows the Tafel polarization curves for uncoated mild steel, coated with PPy and coated with $\mathrm{PPy}+2 \mathrm{D}-\mathrm{MoS}_{2}$

Corrosion rates of mild steel, PPy coated MS and PPy $+2 \mathrm{D}-\mathrm{MoS}_{2}$ coated Mild Steel were measured with the Tafel polarization method to be 15.55, 1.72 and 0.28 respectively. As seen in the Table I, the increase

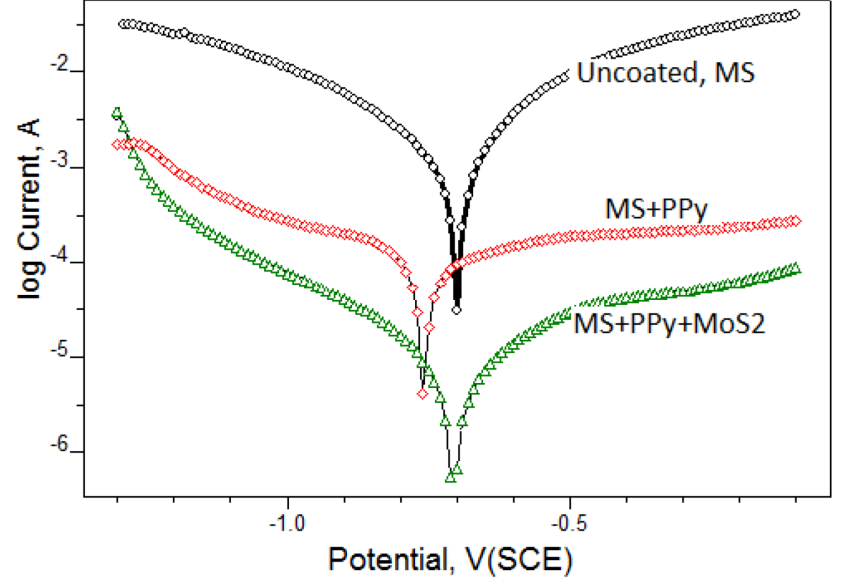

Fig. 2. Tafel polarization curves for MS uncoated and coated with PPy and PPy $+\mathrm{MoS}_{2}$.

TABLE I

Tafel polarization parameters and coating efficiency for the corrosion of MS in $0.1 \mathrm{M} \mathrm{H}_{2} \mathrm{SO}_{4}$.

\begin{tabular}{l|c|c|c|c}
\hline \hline & $\begin{array}{c}-E_{\text {cor }} \\
{[\mathrm{V}]}\end{array}$ & $\begin{array}{c}R_{p} \\
{[\Omega]}\end{array}$ & $\begin{array}{c}\text { Corrosion } \\
\text { rate } \\
{[\text { mm/year }]}\end{array}$ & $\begin{array}{c}\text { Efficiency } \\
{[\%]}\end{array}$ \\
\hline $\mathrm{MS}$ & 0.7126 & 45.3 & 15.55 & - \\
$\mathrm{MS}+\mathrm{PAN}$ & 0.6593 & 51.9 & 12.20 & 21.5 \\
$\mathrm{MS}+\mathrm{PPy}$ & 0.7705 & 983.4 & 1.72 & 88.9 \\
$\mathrm{MS}+\mathrm{PAN}+2 \mathrm{D}-\mathrm{MoS}_{2}$ & 0.6751 & 104.9 & 5.48 & 64.8 \\
$\mathrm{MS}+\mathrm{PPy}+2 \mathrm{D}-\mathrm{MoS}_{2}$ & 0.6718 & 8584 & 0.28 & 98.2
\end{tabular}

in the polarization resistance $\left(R_{p}\right)$ values indicates that the coating formed on the steel surface protects metal by generating a resistance against corrosion. Figure 2 shows that significant protection from corrosion has been achieved with PPy coating. This protection is further enhanced by $2 \mathrm{D}-\mathrm{MoS}_{2}+$ PPy coating. PPy coating protects $88.9 \%$ MS from corrosion, while PPy $+2 \mathrm{D}-\mathrm{MoS}_{2}$ protect $98.2 \%$.

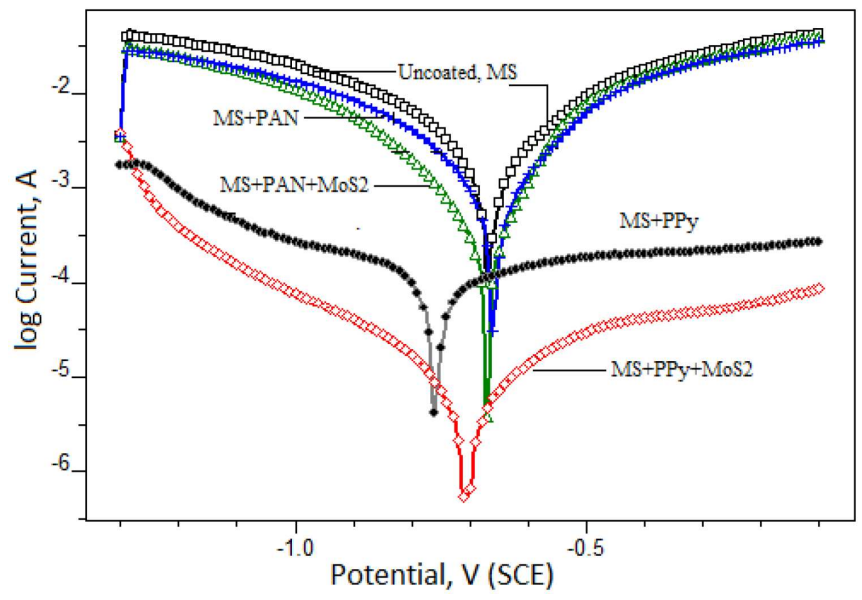

Fig. 3. Tafel polarization curves for MS uncoated and coated with additives. 

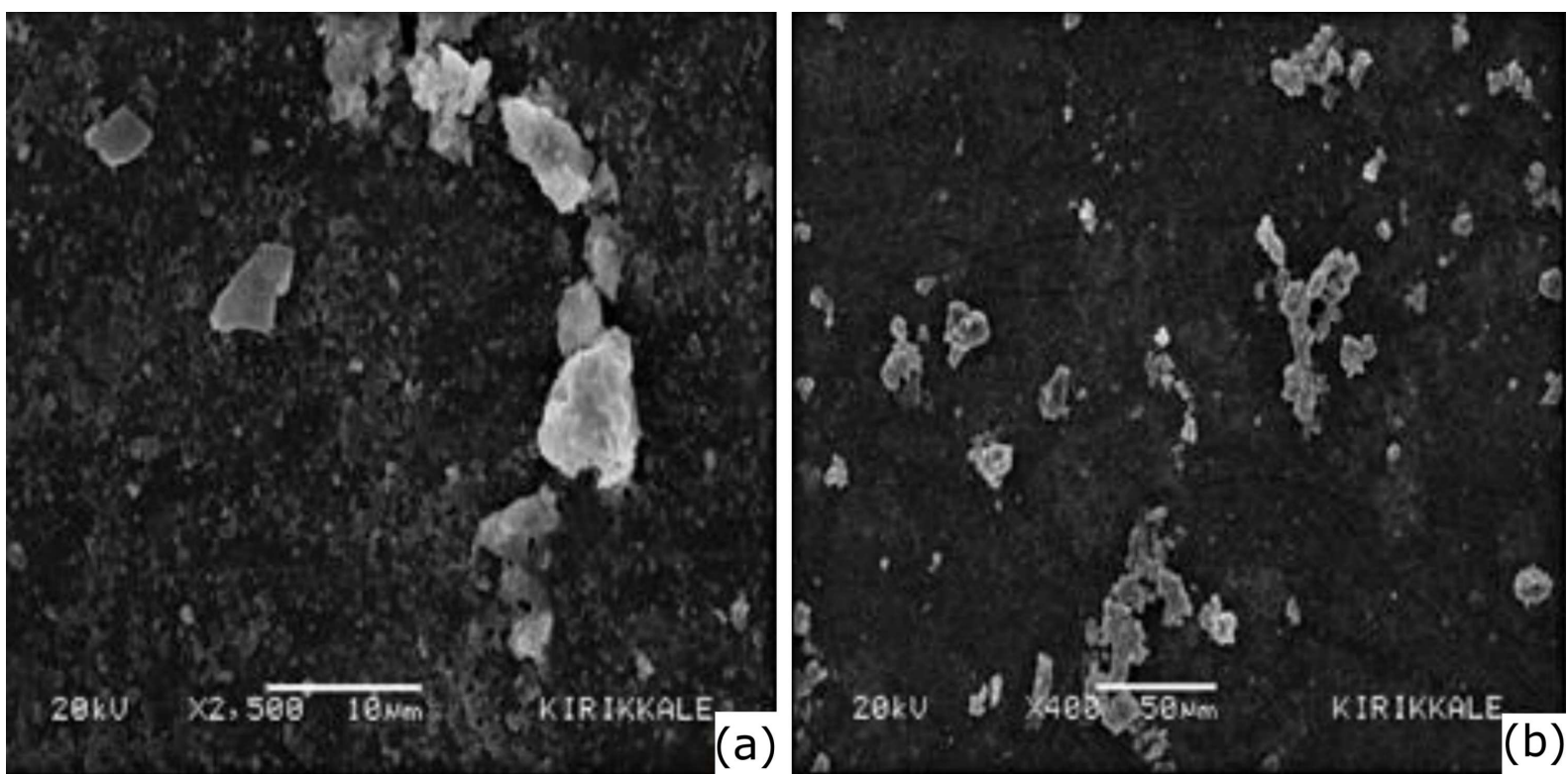

Fig. 4. SEM photographs of the coatings: (a) PAN and (b) PPy.
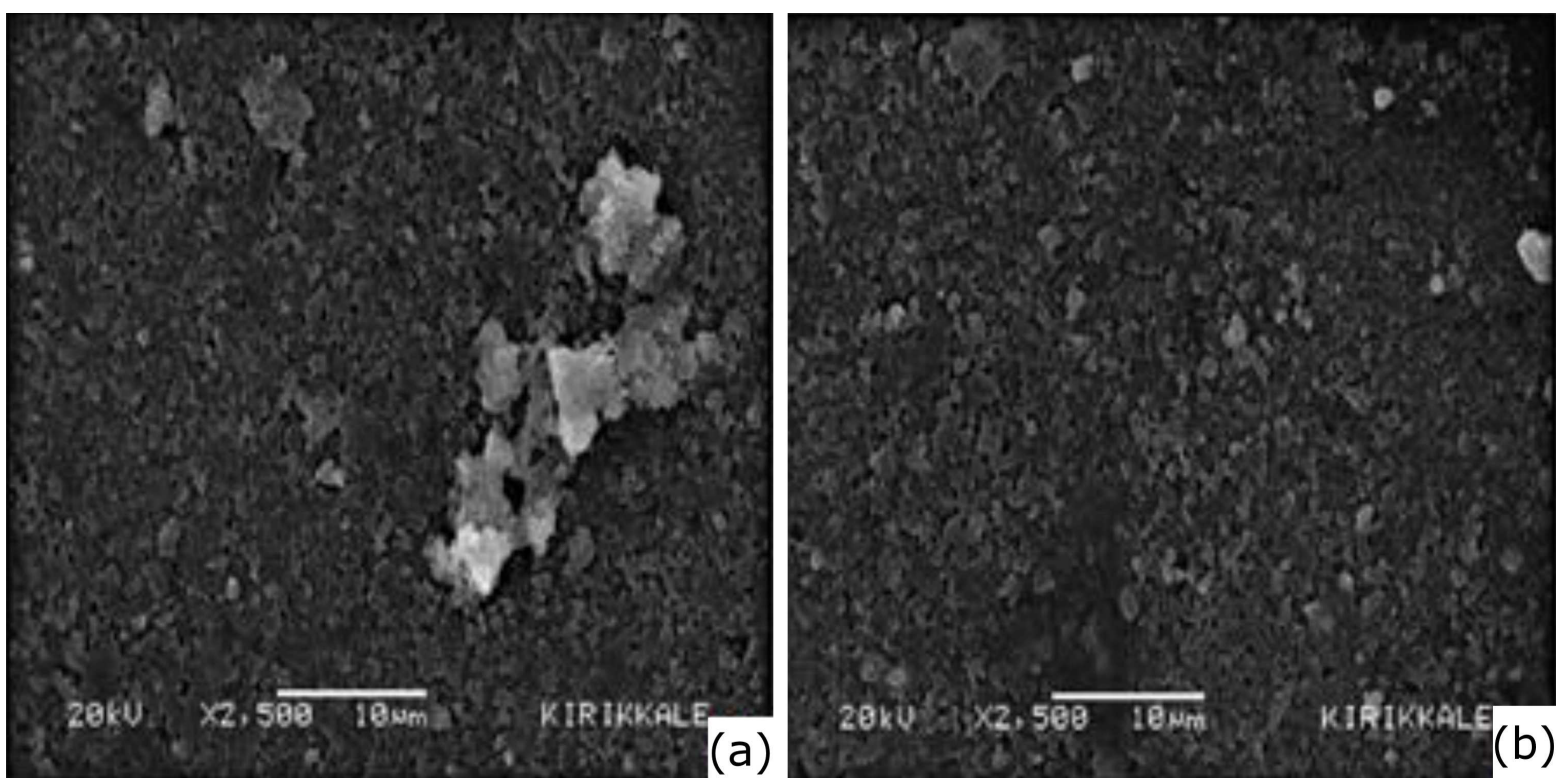

Fig. 5. SEM photographs of the coatings: (a) PAN $+2 \mathrm{D}-\mathrm{MoS}_{2}$, (b) PPy $+2 \mathrm{D}-\mathrm{MoS}_{2}$.

Figure 3 shows the Tafel polarization curves for uncoated MS and mild steel coated with PPy, PPy $+2 \mathrm{D}-$ $\mathrm{MoS}_{2}, \mathrm{PAN}, \mathrm{PAN}+2 \mathrm{D}-\mathrm{MoS}_{2}$.

The corrosion performance of PAN, PPy, PAN doped with 2D-MoS ${ }_{2}$ and PPy doped with 2D-MoS 2 coated on MS was summarized in Table I.

SEM images of the coatings are given in Figs. 4 and 5. In Fig. 4a and $\mathrm{b}$ MS coated with PAN and MS coated with PPy are are represented, respectively. Figure 5a and b shows that coatings doped 2D$\mathrm{MoS}_{2}$ for PAN and PPy more homogeneous than PAN and PPy coatings.

\section{Conclusion}

PPy performance is better because PPy has a more stable layer on the steel surface than PAN. This phenomenon can be explained by the lower potential polymerization of PPy than PAN. The 2D-MoS 2 contribution improves the performance of both $\mathrm{PPy}$ and PAN coating. It may have been an auxiliary agent for good adhesion and coating between MS surface and PPy or PAN coatings. The best protection against corrosion is provided by $\mathrm{PPy}+2 \mathrm{D}-\mathrm{MoS}_{2}$ coating while the lowest performing coating is $\mathrm{PAN}$. The presence of $2 \mathrm{D}-\mathrm{MoS}_{2}$ in coatings 
made of conductive polymers such as polypyrrole and polyaniline on the steel surface is more resistant to steel corrosion. It can also be assumed that 2D-MoS 2 , which has recently been used to reduce friction, has a positive effect on corrosion.

\section{References}

[1] G. Mengoli, M.T. Munari, P. Bianco, M.M. Musiani, J. Appl. Polym. Sci. 12, 4247 (1981).

[2] D.W. DeBerry, J. Electrochem. Soc. 132, 1022 (1985).

[3] K.K. Kanazawa, A.F. Diaz, R.H. Geiss, W.D. Gill, J.F. Kwak, J.A. Logan, J.F. Rabolt, G.B. Street, Chem. Soc. Chem. Commun 854-855 (1979).
[4] A.F. Diaz, Chem. Scr. 17, 142 (1981).

[5] A.G. Macdiarmid, Jin-Chih Chiang, M. Halpern, WuSong Huang, Shao-Lin Mu, L.D. Nanaxakkara, Somasiri Wanqun Wu, S.I. Yaniger Cryst. Liq. Cryst. 121, 173 (1985).

[6] T.J. Pan, X.W. Zuo, T. Wang, J. Hu, Z.D. Chen, Y.J. Ren, Journal of Power Sources, 302, 180 (2016).

[7] A. Asan, M. Kabasakaloglu, M.L. Aksu, Elektrokhimiya, 41, 175 (2005)

[8] M. Bazzaoui, L. Martins, E.A. Bazzaoui, J.I. Martins, Electrochim. Acta 47, 2953 (2002).

[9] M. Fahlman, S. Jasty, A.J. Epstein, Synthetic Metals 85, 1323 (1997).

[10] G. Asan, H. Çelikkan, GUMMFD 32, 617 (2017). 